\section{WHAT IS THE CULPRIT IN CORONARY ANOMALIES?}

\section{To the Editor:}

I read with great interest and excitement the recent report by Bartoli and colleagues ${ }^{1}$ concerning the mechanism of ischemia in anomalous origination of a coronary artery. Anomalous left coronary artery arising from the opposite sinus of Valsalva (called L-ACAOS when the involved artery has an intramural course $^{2}$ ) is one of the most common causes of sudden cardiac death in the young, as observed in United States military recruits (mean age of 19 years). ${ }^{3}$

The true cause and individual case severity of anomalous coronary origination have been the elusive "holy grail" sought by many investigators. This search has been persistently marred by inadequate evidence and frustrating uncertainty on clinical grounds. The report of a calf with such an anomaly, studied by expert investigators with amazing means and dedication, is therefore a welcome event. Bartoli and colleagues ${ }^{1}$ hypothesized that ischemia (at least during their experiments) was "present and caused by the course of this anomalous artery in the space between the aorta and pulmonary artery." Aortography is not a proper method for studying the coronary course in this location, however, and ischemia was not well demonstrated by Bartoli and colleagues' improvised protocol in the animal laboratory (aimed at

\footnotetext{
The Editor welcomes submissions for possible publication in the Letters to the Editor section that consist of commentary on an article published in the Journal or other relevant issues. Authors should: • Include no more than 500 words of text, three authors, and five references. - Type with double-spacing. - See http://jtcs.ctsnetjournals.org/misc/ifora.shtml for detailed submission instructions. - Submit the letter electronically via jtcvs.editorialmanager.com. Letters commenting on an article published in the JTCVS will be considered if they are received within 6 weeks of the time the article was published. Authors of the article being commented on will be given an opportunity of offer a timely response ( 2 weeks) to the letter. Authors of letters will be notified that the letter has been received. Unpublished letters cannot be returned.
}

documenting the flow reserve but not ischemia). Electrocardiography is not an acceptable or quantifiable marker of ischemia (in Figure 1 of Bartoli and colleagues' report, ${ }^{1}$ the $\mathrm{T}$ wave seems to be negative in the 3 tracings, in the absence of ST-segment depression). Additionally, the current prevailing opinion is that the cause of ischemia in this type of coronary anomaly (L-ACAOS or the similar entity R-ACAOS) is best studied with intravascular ultrasonography, which can satisfactorily document in vivo the severity of obstruction at baseline or during exercise. ${ }^{4,5}$ Flow velocity is an interesting means of evaluation, but flow cannot be quantified unless accompanied by appropriate data concerning the variable baseline and exercise-induced cross-sectional area of stenosis.

Bartoli and colleagues ${ }^{1}$ are to be congratulated for their admirable attempt at taking a new approach to this fascinating subject. It seems to me, however, that clarifying the pathology requires study of a large clinical population that has been properly evaluated for severity of obstruction-correlated with symptomsand for empirical response to the elimination of intramural stenosis (by means of stent angioplasty or surgical correction).

My group at the Texas Heart Institute is completing a multiyear research study of a large case series (65 patients with R-ACAOS), and we hope that our findings will substantially advance current knowledge in this field.

\section{Paolo Angelini, MD \\ The Center for Coronary Artery Anomalies \\ Texas Heart Institute Houston, Tex}

\section{References}

1. Bartoli CR, Wead WB, Giridharan GA, Prabhu SD, Koenig SC, Dowling RD. Mechanism of myocardial ischemia with anomalous left coronary artery from the right sinus of Valsalva. $J$ Thorac Cardiovasc Surg. 2012;144:402-8.

2. Angelini P, Flamm SD. Newer concepts for imaging anomalous aortic origin of the coronary arteries in adults. Catheter Cardiovasc Interv. 2007;69: 942-54

3. Eckart RE, Scoville SL, Campbell CL, Shry EA, Stajduhar KC, Potter RN, et al. Sudden death in young adults: a 25-year review of autopsies in military recruits. Ann Intern Med. 2004;141:829-34.

4. Angelini P. Coronary anomalies and sports activities. In: Lawless CE, ed. Sports Cardiology Essentials: Evaluation, Management and Case Studies. New York: Springer; 2010:277-98.

5. Angelini P, Walmsley RP, Libreros A, Ott DA. Symptomatic anomalous origination of the left coronary artery from the opposite sinus of Valsalva Clinical presentations, diagnosis, and surgical repair. Tex Heart Inst J. 2006;33:171-9.

\section{http://dx.doi.org/10.1016/}

j.jtcvs.2012.06.060

\section{Reply to the Editor:}

We thank Dr Angelini for his kind review of our work. We read his letter with great interest and welcome the opportunity to clarify a few methodological points. Each of his comments is addressed below.

Until now, the mechanism of myocardial ischemia with an anomalous coronary artery represented an important and elusive milestone in congenital cardiology. Our findings demonstrated in a calf that an ectopic left coronary artery that coursed from the right sinus of Valsalva between the aorta and the pulmonary trunk produced myocardial ischemia and ischemic myocardial remodeling. ${ }^{1}$ As cardiac metabolic demands increased, compression between the great vessels increased coronary resistance and decreased coronary blood flow. These findings provide insight into a potential mechanism of myocardial ischemia when a coronary artery courses between the great vessels and raise additional questions. To further define the clinical implications, additional studies are needed.

We agree that aortography is an inappropriate technique to study coronary anomalies. Indeed, in our study the anomaly was surreptitiously identified via aortogram during a routine coronary microembolization procedure to create ischemic heart failure in a calf. The animal was not used 